Original Research Article

\title{
Influence of interventions on prescription writing practices in a tertiary care teaching hospital
}

\author{
Priti P. Dhande ${ }^{1 *}$, S. D. Kawade ${ }^{2}$
}

${ }^{1}$ Department of Pharmacology, Bharati Vidyapeeth Deemed to be University Medical College, Pune, Dhankawadi,

Maharashtra, India

${ }^{2}$ Department of Quality Bharati Hospital and Research Centre, India, Dhankawadi, Pune, Maharashtra, India

Received: 30 September 2019

Revised: 12 November 2019

Accepted: 14 November 2019

\section{*Correspondence to: \\ Dr. Priti Dhande, \\ Email: pritidhande76@ \\ gmail.com}

Copyright: (C) the author(s), publisher and licensee Medip Academy. This is an openaccess article distributed under the terms of the Creative Commons Attribution NonCommercial License, which permits unrestricted noncommercial use, distribution, and reproduction in any medium, provided the original work is properly cited.

\begin{abstract}
Background: Prescription practices should be good so that they are correctly interpreted and medications are used or administered accordingly. Studies are found usually focusing on WHO prescribing indicators in various healthcare set-ups. This prescription audit study was conducted to analyse the prescription practices in a tertiary care hospital and see the influence of various interventions on these practices.

Methods: It was a quality initiative undertaken to retrospectively assess and evaluate the change in the prescription writing practices in the in-patient department of a tertiary care teaching hospital from March 2017 to April 2018. Number of interventions were conducted to meet the compliance of prescription parameters during the study period and prescription parameters was analysed again thereafter for one-year to see the change.

Results: An overall increase in compliance of prescription parameters was found from $67.3 \%$ in March 2017 to $94.8 \%$ in June 2017. Thereafter this was consistently maintained upto $91 \%$ during the study period. Most of the prescription parameters were seen to be improved like allergy history taking from 52.2-95.6\%; mention of prescriber's name, sign, registration number from $53.5-86 \%$; consideration to food-drug interaction from $28.7-99.5 \%$.

Conclusions: Prescription writing practices were quite poor initially which improved after various interventions were carried out in the study area.
\end{abstract}

Keywords: Prescription, Audit, Interventions, Improvement

\section{INTRODUCTION}

Prescription writing is a skill which invites accuracy in the process to ensure the appropriateness of the message to be passed to the pharmacist, nurse or patient for whom it is meant. Pharmacists and patients are the addressee of the prescription when it is given in out-patient department while drug prescriptions on medication charts are instructions for the nurses to respond accordingly. The completeness and legibility of the prescription is very crucial for its correct interpretation and administration (or consumption) of medication without any errors.

Medication errors are very common in day-to-day clinical practice and are avoidable with proper targeted interventions which can improve healthcare. To achieve this, we have to focus on the cause and frequency of medication errors in clinical settings and what allows them to slip in different scenarios of patient management. Similar initiative was undertaken in the western world (U.K, U.S.A) with success. ${ }^{1}$ 
Studies conducted in countries like Australia, Canada and Argentina have evaluated the impact of educational interventions on prescribing errors using an audit of medication charts pre and post intervention. ${ }^{2-4}$ Indian authors have mainly focused on studying the WHO prescribing indicators in various setups like secondary level government hospitals, tertiary care hospitals, private hospitals or among general practitioners. ${ }^{1-3}$

Only one study carried out at out-patient department of a multispecialty hospital in western India has mentioned the other aspects of prescription like legibility, complete drug details for treatment (name, dose, route, frequency, duration), drug duplication, drug-drug and food-drug interactions, physicians name, sign and registration number, etc. But this study was a cross sectional study scrutinizing the prescriptions by an audit process. ${ }^{3}$

On extensive literature search, no study was found conducted in Indian hospital setup which has assessed the effect of interventions on such prescription parameters. Hence, this retrospective study was planned to analyse the prescriptions issued by the doctors in the in-patient department of a tertiary care hospital in western India and using prescription auditing as a tool for our targeted interventions we have compared the pre-intervention and post-intervention status of prescription writing in the hospital.

\section{METHODS}

This was a quality initiative undertaken to retrospectively assess and evaluate the improvisation in the prescription writing practices of the in-patient areas in Bharati Hospital and Research Centre, Pune which is a tertiary care teaching hospital. The medication charts of inpatients from their case files were surveyed for data collection from March 2017 to April 2018. Sample number of files to be reviewed for this purpose was based on the bed occupancy of the hospital and accordingly calculated every month which came to about $35-40 \%$ coverage of ward occupancies and $100 \%$ of intensive care unit occupancies.

The prescription parameters studied were legibility of prescription, completeness of drug details (name in capitals, dose, route and frequency of administration), drug duplication, use of approved abbreviations, consideration given to drug-drug and food-drug interactions, adherence to antimicrobial policy and hospital drug formulary and authorization signature with registration number of prescriber.

As a part of quality initiatives, a number of interventions were conducted to meet the compliance of prescription parameters according to National Accreditation Board for Hospitals (NABH) standards. These interventions included power point based educational lectures for resident doctors and consultants directed towards necessary parameters in prescription and their clinical as well as legal importance, explanation about use of approved abbreviations while prescribing (Tab for tablet, $\mathrm{ml}$ instead of cc, unit in place of IU, etc.,) and avoiding dangerous abbreviations which could cause medication errors, adherence to hospital's Antimicrobial policy and Drug formulary by prescribers was also stressed upon during the lectures, ideal prescription template was prepared and communicated to the prescribers and list of common drug-drug and food-drug interactions was prepared and prescribers were made aware of these interactions.

After a series of such interventions in groups of resident doctors and consultants separately as well as at individual departmental levels, the compliance to prescription parameters was analysed again and continuously thereafter for one-year period to see the change and its consistency.

\section{Data analysis}

Data were entered in Microsoft Excel and then presented in percentages after analysis.

\section{RESULTS}

Every month a sample of prescriptions were audited for analysing the compliance to prescription parameters. Number of files to be reviewed for this purpose was based on the bed occupancy of the hospital and accordingly calculated every month which came to about $35-40 \%$ of ward patients and $100 \%$ of intensive care unit patients. So, averagely 400 prescriptions were audited every month for the study.

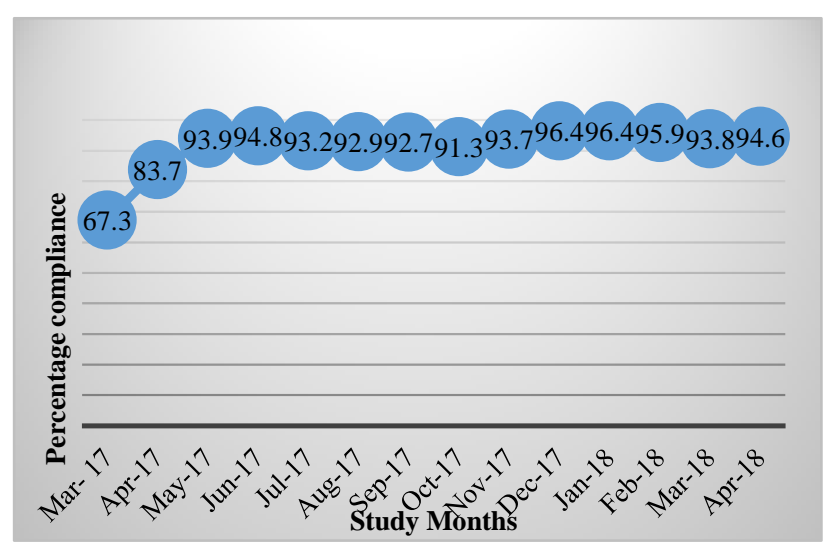

\section{Figure 1: Hospital wide compliance of total prescription parameters in percentage.}

Figure 1 shows an initial increase in compliance of prescription parameters from 67.3-94.8\% (March 2017 to June 2017) and then consistency in the compliance above and around $91 \%$ is seen in the following months till April 2018.

Figure 2 depicts the improvement seen in various prescription parameters at the beginning (March 2017) 
and end of the study period (April 2018). Total change in these improved parameters is seen to be increasing from $59.82-92.62 \%$. Drastic change was observed in allergy history taking from 52.2-95.6\%; mention of prescriber's name, sign, registration number from $53.5 \%$ to $86 \%$; consideration to food-drug interaction from $28.7-99.5 \%$, etc. Drug name to be written in capitals was improved from $62.7 \%$ in March 2017 to $97.1 \%$ in April 2018.

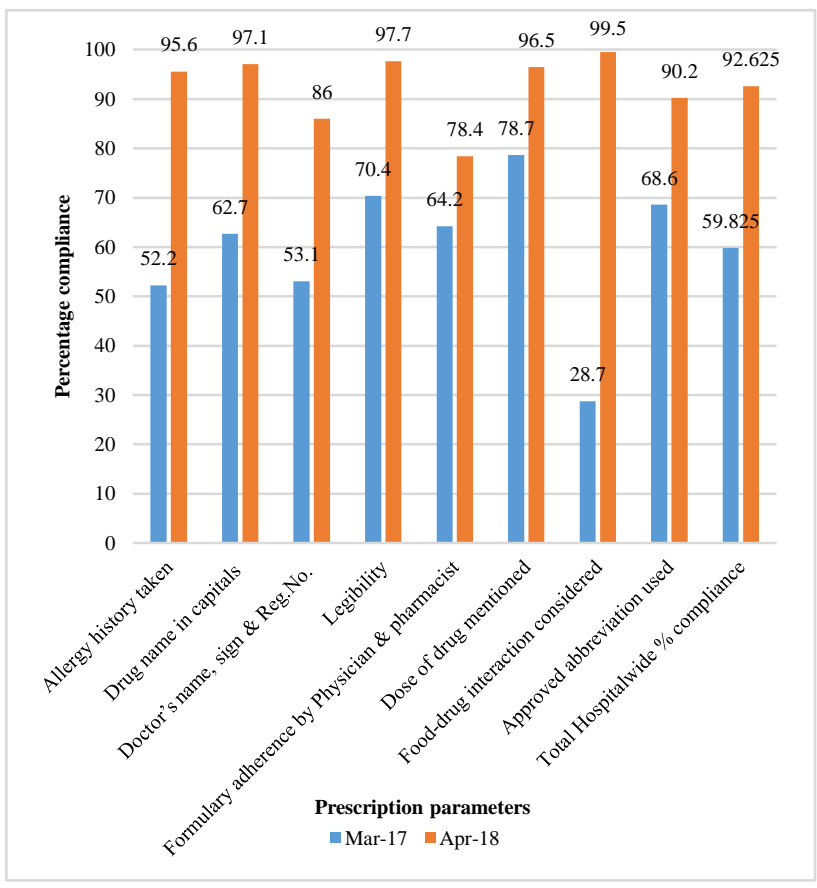

Figure 2: Change in the prescription parameters over the study period.

\section{DISCUSSION}

This study was carried out to analyse the effect of various interventions on prescription writing practices in a tertiary care teaching hospital.

On analysing the compliance of prescription parameters (according to $\mathrm{NABH}$ standards) in the initial study period, it was found to be $67.3 \%$ for total parameters. There was a need to instigate certain measures for improvising the prescription quality at the study site. Educational sessions were conducted to convey the importance of completeness of prescription parameters clinically as well as legal value of some parameters like date of prescription and sign and registration number of doctor was conveyed to the participants. Ideal prescription template was displayed in all outpatient departments and wards for the prescribers to refer and imply in their practice.

Legibility of prescription is very important for proper message to be conveyed to the dispensing pharmacist, nursing staff or patient so that medication errors can be prevented. Authors have reported that the legibility of doctor's handwriting is the worst among all healthcare professionals. ${ }^{3,4}$ In the initial months of the study period, this parameter of legibility was nearly $70 \%$. With the mandatory writing of drug name in capitals and urge to mention prescription parameters clearly and completely, the legibility also improved to $97.7 \%$ by the end of the study.

Efforts were taken to scrutinize each prescription parameter and initiate measures to improve the compliance of these parameters. Allergy history taking and/or noting it down in the patient's file was not a routine practice by all prescribers in the study hospital for every patient. Considering the seriousness of risk, it may pose if a patient is inappropriately given a drug he/she is allergic to, noting of allergy history in the patient's case file was made mandatory. Inspite of this when it was found that allergy history was not considered, a special column was printed on the initial assessment form in patient case files to note down their allergy history and then this parameter was followed for its compliance in the further study period.

In private clinics or hospitals, physicians write prescriptions where they use medical abbreviations, but in large teaching hospitals, this job is of the medical and surgical residents who are tasked with transcribing the drug orders on the medication chart for the nurses to follow. Use of unapproved or dangerous medical abbreviations can result in miscommunication because the other healthcare staff reading and processing these medication orders may not be familiar with these abbreviations. Such circumstances can result in medication errors in dispensing or administration of a medication and pose a great risk to the patient's health or even life. At the study site many such dangerous abbreviations were being used initially ( $U$ for units, $\mathrm{T}$ for tablets, $\mathrm{C}$ for capsules, IU for international units, $\mathrm{cc}$ in place of millilitres or $\mathrm{ml}$, etc). When this was discovered during the audit, all the participants were made aware of the risks associated with use of such dangerous abbreviations giving appropriate examples and list of dangerous abbreviations was prepared and displayed in patient care areas to be avoided by prescribers. At the same time which abbreviations should be used to depict metrics or medical terminologies were also informed and then further audited continuously. Rampant or common use of any particular abbreviation if noted down in any specific department was brought to the notice and measures instituted accordingly to train the prescribers in that department for use of approved and safe abbreviations.

As per the NABH requirements, a hospital drug formulary was prepared and the prescribers trained about the concept of this formulary and its use. When the adherence to formulary was analysed, it was only $64 \%$. To improve this adherence, hard copy of hospital drug formulary was made available at all patient care areas while PDF soft copy was given to each prescriber on his/her mobile or laptop according to convenience. A 
minor improvement was observed in the formulary adherence from $64.2-78.4 \%$. The culture of prescribers to choose their favourite brands and ways to remember their names, resistance and lack of enough time to refer the drug formulary were the major hurdles responsible for a poor rise in this parameter inspite of interventions.

The prescription parameters given least consideration in the initial study period were drug-drug $(33.1 \%)$ and fooddrug $(28.7 \%)$ interactions. Educational sessions on commonly encountered and possible drug-drug and drugfood interactions were planned and the participants were explained about ways to minimize such interactions. During the further months of the study, both these parameters were gradually seen to be improved to above $98 \%$ by the end of the study.

Initially overall compliance to prescription parameters was assessed and then to find out the non-compliant prescribers, ward wise and departmental unit-wise analysis was conducted. Feedback was provided to the respective departmental heads where non-compliance of prescription parameters was observed in their departmental units. Later on even the names of noncomplaint resident doctors were communicated to the incharges so that they could be followed up and supervised for their prescription practices after getting education on appropriate prescribing.

\section{CONCLUSION}

Prescription writing practices were quite poor in the study set-up initially specially for those parameters which could lead to serious consequences on misreading. Interventions were found to be useful in improving these prescription parameters and prescribers were consistently following the ideal prescription guidelines to avoid medication errors of prescribing type.

Funding: No funding sources Conflict of interest: None declared

Ethical approval: The study was approved by the Institutional Ethics Committee

\section{REFERENCES}

1. Dean B, Schachter M, Vincent C, Barber N. Causes of prescribing errors in hospital inpatients: a prospective study. Lancet. 2002;359(9315):1373-8.
2. Shaw J, Harris P, Keogh G, Graudins L, Perks E, Thomas PS. Error reduction: academic detailing as a method to reduce incorrect prescriptions. Eur J Clin Pharmacol. 2003;59:697-9.

3. Kozer E, Scolnik D, Macpherson A, Rauchwerger D, Koren G. The effect of a short tutorial on the incidence of prescribing errors in pediatric emergency care. Can J Clin Pharmacol. 2006;13:e285-91.

4. Otero P, Leyton A, Mariani G, Cernadas CJM. Medication errors in pediatric inpatients: prevalence and results of a prevention program. Pediatric. 2008;122:e737-43.

5. Potharaju HR, Kabra SG. Prescription audit of outpatient attendees of secondary level government hospitals in Maharashtra. Indian $\mathrm{J}$ Pharmacol. 2011;43(2):150-6.

6. Sharma M, Sanneving L, Mahadik K, Santacatterina M, Dhaneria S, Lundborg CS. Antibiotic prescribing in women during and after delivery in a non-teaching, tertiary care hospital in Ujjain, India: a prospective cross-sectional study. J Pharm Policy Pract. 2013;6:9.

7. Shelat PR, Kumbar SK. Analysis of outdoor patients' prescriptions according to World Health Organization (WHO) prescribing indicators among private hospitals in Western India. J Clin Diagn Res. 2015;9(3):FC01-4.

8. Gopalakrishnan S, Ganeshkumar P, Katta A. Assessment of prescribing practices among urban and rural general practitioners in Tamil Nadu. Indian $\mathbf{J}$ Pharmacol. 2013;45(3):252-7.

9. Solanki ND, Shah C. Prescription audit in outpatient department of multispecialty hospital in western India: an observational study. Int $\mathbf{J}$ Clin Trials. 2015;2:14-9.

10. Lyons R, Payne C, McCabe M, Fielder C. Legibility of doctors' handwriting: quantitative comparative study. BMJ. 1998;317:863-4.

11. Berwick DM, Winickoff DE. The truth about doctors' handwriting: a prospective study. BMJ. 1996;313:1657-8.

Cite this article as: Dhande PP, Kawade SD. Influence of interventions on prescription writing practices in a tertiary care teaching hospital. Int $\mathbf{J}$ Basic Clin Pharmacol 2019;8:2685-8. 